\title{
CFD BASED PROCESS MODELLING OF A ROTARY FURNACE FOR ALUMINIUM SCRAP MELTING
}

\author{
Bo ZHOU ${ }^{1}$, Yongxiang YANG ${ }^{1}$, Markus A. REUTER ${ }^{1}$, Udo M.J. BOIN ${ }^{1}$ \\ ${ }^{1}$ Delft University of Technology, Department of Applied Earth Sciences, \\ Mijnbouwstraat 120, 2628 RX Delft, The Netherlands
}

\begin{abstract}
A computational fluid dynamics (CFD) based process model of a rotary furnace for aluminium scrap melting was developed and integrated with user-developed submodels. The model consists of a gas region with turbulent flow and combustion, radiative heat transfer in the upper part of the furnace, a solid region of the furnace lining, and a solid-liquid region of salt and metal in the lower part of the furnace. The solid-liquid region, where scrap melting and burn-off reactions take place, was regarded as a conducting solid. Scrap phase change (melting) and scrap burn-off was simulated by the user-developed submodels. A descritized population balance model (PBM) for aluminium scrap was established to represent the distributed nature of the scrap feed. The melting behaviour of aluminium scrap was simulated with the exchange of information between the melting sub-model and the CFD calculations. Simulations of the melting process were made to model the flow and thermal phenomena in such a furnace. Case studies, such as the influence of the scrap size, shape and quality, as well as burn-off rate, were carried out.
\end{abstract}

KEYWORDS: computational fluid dynamics (CFD); process modelling; aluminium scrap melting; population balance model; rotary furnace

\section{NOMENCLATURE}

$C_{\text {void }}$ voidage coefficient for effective thermal conductivity

$C_{\text {rot }}$ rotation coefficient for effective thermal conductivity

$f$ mass fraction in scrap-salt zone

$h$ heat transfer coefficient

$\Delta H$ latent heat

$r$ radius

$t$ time

$T$ temperature

$T_{f} \quad$ bulk melt temperature

$\delta_{g} \quad$ voidage of the scrap-salt zone

$\lambda_{\text {mix }}$ thermal conductivity of the mixture in scrap-salt zone

$\lambda_{\text {eff }}$ effective thermal conductivity in scrap-salt zone

$\rho_{p} \quad$ density of the particle

\section{Subscripts}

$m, p$ melting point of the particle

$m, l$ metal in liquid state in scrap-salt zone

$m, s$ metal in solid state in scrap-salt zone

$s, l \quad$ salt in liquid state in scrap-salt zone

$s, s$ salt in solid state in scrap-salt zone

\section{INTRODUCTION}

Secondary aluminium production is increasing very rapidly in recent years and it will keep a steady growing in the future (European Aluminium Association). Recycling is a critical component of the aluminium industry based on its favourable economic impact on production and its contribution to environment. During secondary aluminium recovery, aluminium scraps are processed often in a rotary melting furnace in Europe, where it functions simultaneously as a smelter and a phase separator (Aluminium Handbook Vol. 2, 2003). Figure 1 illustrates the complex phenomena in such a furnace. The scrap feed is charged into a rotary furnace, melting, mixing and being cleaned under a salt flux layer. The rotary furnace is normally operated at a temperature around $800^{\circ} \mathrm{C}$. Natural gas combustion with oxygen is used as the main energy source. The produced liquid aluminium is tapped into a holding furnace, further refined and then directly transported to the industrial partners or cast into ingots. The salt slag with various contaminations should be further processed and reused.
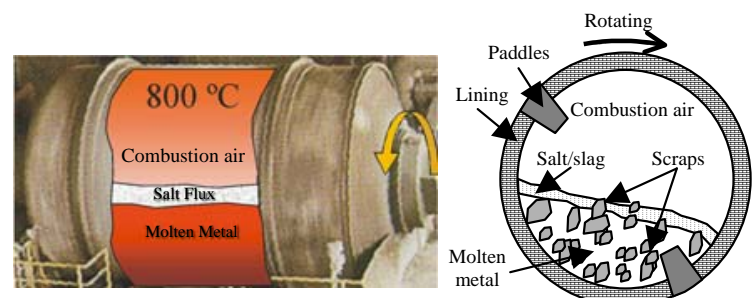

Figure 1: Illustration of the rotary melting furnace and the complex inside phenomena.

Though the process for reclamation of aluminium scrap has been developed for many years, little public knowledge is available. The complexity in the process is due not only to the high temperature effect and complex chemical reactions, but also to the highly complex scrap feed with a distributed nature of aluminium types, sizes, shapes, compositions, paintings and other contaminations. The objective of this study is to develop a computational fluid dynamics (CFD) based process model, predicting the melting rate and energy distribution in relation to the melting behaviour of different scrap types and properties, and to make improvements of the process based on the model results.

This work was started from the experimental study (Zhou et al., 2002) and the numerical modelling (Zhou et al., 2003) of aluminium particle melting in molten melts. 
Then a CFD based process model was developed based on a commercial CFD package, ANSYS-CFX 5.6 (ANSYSCFX, 2003), and integrated with user-developed submodels. Turbulent fluid flow, gas combustion, radiation, and conjugated heat transfer were simulated in the CFD framework. To represent the distributed nature of the aluminium scrap feed with different types, sizes, shapes etc., a melting sub-model with population balance modelling (PBM) (Sohn, et al, 1979) was developed and integrated into the CFD framework. Furthermore an aluminium burn-off sub-model was developed and integrated to take into account the heat generated due to the scrap burn-off (oxidation) effect during the melting process. Industrial observations and data measurements were performed on the purpose of model development and calibration. Finally a number of case studies were conducted based on the CFD based process model and the user-developed sub-models.

\section{CFD BASED PROCESS MODEL}

\section{General information of the CFD based process model}

CFD as a research tool was found useful in studying various metallurgical processes, where momentum, heat and mass transport play important roles in reaction kinetics and reactor performance. In this study, a commercial CFD package, ANSYS-CFX 5.6 (ANSYSCFX, 2003), was used as a framework of the process model, coupled with user-developed sub-models.

The industrial scale rotary furnace is $3.0 \mathrm{~m}$ in inner diameter, $3.65 \mathrm{~m}$ in outer diameter including the lining structure and $6.9 \mathrm{~m}$ in length. As shown in Figure 2, the model consists of a gas region with turbulent flow, combustion, and radiative heat transfer in the upper part of the furnace, a solid region of the furnace lining, and a solid-liquid region of salt and aluminium metal in the lower part of the furnace. The rotation of the furnace, about $1.33 \mathrm{rpm}$, and the agitation of the paddles built in the furnace wall were not included in this model.

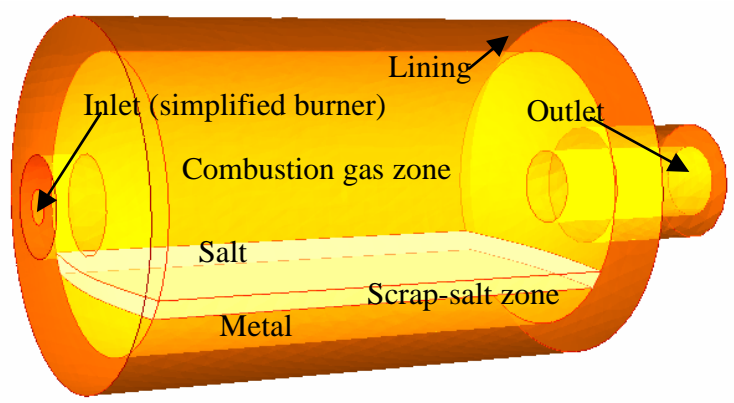

Figure 2: Geometry and boundaries of the furnace

An unstructured mesh, as shown in Figure 3, was applied in this study, the total number of the meshes is 88566 in this case and finer meshes were used in some sensitive areas, e.g. burner and flame areas, while coarser meshes were applied in the end part of the furnace and in the furnace lining. The CPU time for a whole furnace cycle lasting about 16200 s, on a Pentium IV, $2.66 \mathrm{GHz}$ PC, is about 35 hours with the time step set at 30 seconds (for the first 300 seconds, smaller time steps were used to get a stable flow and temperature field, it gradually increases from $0.1 \mathrm{~s}$ to $30 \mathrm{~s}$ ). Mesh independence test and time step independence test have been carried out. Three mesh configurations (88566, 178635 and 269508 elements, approximately 1: 2: 3), and time step configurations (60s, $30 \mathrm{~s}, 10 \mathrm{~s}, 3 \mathrm{~s}$ and $1 \mathrm{~s}$ ) were tested. The result indicates that grid and time step independent results can be obtained.

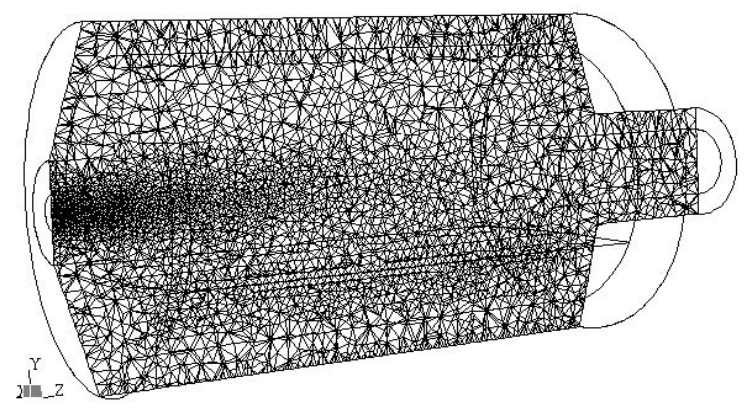

Figure 3: Mesh configuration on the centre plane $(x=0)$

\section{Treatment of the scrap zone}

The current CFD process model is a single phase model, where gas flow and combustion in the air zone was simulated, and the scrap-salt zone was regarded as a conducting solid / stagnant liquid, a mixture of scrap and salt solids. Thus the fluid flow in the scrap-salt zone was not considered, while the effect on heat transfer in this zone was represented by a number of thermal parameters. Phase change of scrap and salt was handled by the userdeveloped melting sub-model.

In the scrap-salt zone, it was assumed that scrap and salt are well mixed. The thermal properties of the mixed material were calculated based on the mass fraction and the phase state of the materials in the mixture. The effective density of this region was calculated based on densities of solid scrap and salt, and it were assumed not to change during the process, despite the effect of thermal expansion. Heat capacity was also defined in a similar way. Effective thermal conductivity was calculated based on the expressions below, Equations (1) and (2). Some augmentation coefficients for thermal conductivity were applied to take into account the influence of the voidage in the scrap-salt zone, as well as the effect on heat transfer due to the fluid flow in the scrap-salt zone, agitated by furnace rotation. The influence of the voidage in the scrap-salt zone is represented by a voidage coefficient, $C_{\text {void }}$, and the effect on heat transfer due to furnace rotation is represented by a rotation coefficient, $C_{\text {rot }}$. The values of these parameters were adjusted, and in the present model, $C_{\text {void }}$ was set as 5.0 and $C_{\text {rot }}$ was assumed to be changing between 2.0 and 50.0 depending on the liquid to solid ratio in the scrap-salt zone during the melting process.

$$
\begin{aligned}
\lambda_{\text {mix }}= & \left(\lambda_{m, s} \cdot f_{m, s}+\lambda_{m, l} \cdot f_{m, l}+\right. \\
& \left.\lambda_{s, s} \cdot f_{s, s}+\lambda_{s, l} \cdot f_{s, l}\right) \cdot\left(1-\delta_{g}\right) \\
\lambda_{\text {eff }}= & C_{\text {void }} \cdot C_{\text {rot }} \cdot \lambda_{\text {mix }}
\end{aligned}
$$

\section{Initial conditions}

The initial temperature in the air zone and scrap-salt zone was set as $303 \mathrm{~K}$ in the air zone where the pressure is set 
as $1 \mathrm{~atm}$. The initial temperature in the lining structure was imported from a previous steady simulation of heating the empty furnace. The initial size distribution of the scrap was defined and classified into several scrap groups.

\section{Boundary conditions}

The inlets of the burner for natural gas and oxygen are really small compared to the furnace body. High gradient of velocities and species around the burner area require for small time steps and fine meshes, which is very time consuming. In this case, due to the limit computing power, the inlet was simplified to reduce the computing time. The profile of velocity, temperature and mass flows of gases at the inlet was defined using CEL, based on a previous simulation with a full burner structure and finer meshes. Pressure boundary condition was set for the outlet. Heat transfer coefficient was applied for outside wall. In this case, it's set as $15 \mathrm{~W} / \mathrm{m}^{2} \mathrm{~K}$ and the environmental temperature is set as $303 \mathrm{~K}$. These figures are determined based on static calculations and industrial observations.

\section{Physical models of turbulence, combustion and radiation}

The widely used $k-\varepsilon$ model was applied in most of the simulations in this study, where $k$ is the turbulence kinetic energy and is defined as the variance of the fluctuations in velocity, $\varepsilon$ is the turbulence eddy dissipation, the rate at which the velocity fluctuations dissipate. The $k-\varepsilon$ model has proven to be stable, numerically robust and has a wellestablished regime of predictive capability. However, it's known to have failings for some classes of flows, e.g. recirculation flows. In this research, other turbulence models and parameters, such as RNG $k-\varepsilon$ model, were also tested, while there's only a small influence on the gas flow, combustion and the scrap melting process under the current configuration.

Eddy dissipation model (ANSYS-CFX, 2003) was used for the combustion of the natural gas with oxygen. Because of its simplicity and robust performance in predicting turbulent reacting flows, this model has been widely applied in the prediction of industrial flames. It can be used in a wide range of turbulent reacting flows covering premixed and diffusion flames. This model is based on the concept that chemical reaction is fast relative to the transport processes in the flow. When reactants mix at the molecular level, they instantaneously form products. In turbulent flows, this mixing time is dominated by the eddy properties, and therefore, the rate is proportional to a mixing time defined by the turbulent kinetic energy, $k$, and its dissipation rate, $\varepsilon$. This concept of reaction control is applicable in many industrial combustion problems where reaction rates are fast compared to reactant mixing rates.

For the scrap melting process in the rotary furnace, heat transfer from the gas zone through the gas-scrap interface is the key for the scrap melting process and radiation plays the dominant role. Several radiation models were implemented in ANSYS-CFX 5.6, including P-1, Discrete Transfer Method (DTM) and Monte Carlo Model (MCM). These radiation models were tested and their effect on melting was studied. The result shows that the radiation model only has a small influence on scrap melting, and the total scrap melting time is very close for the simulations with different radiation models and parameters. DTM was chosen in most of the simulations because of its accuracy, robustness and less computing time compared with MCM.

The emissivity of the furnace wall was also tested with the values between 0.7 and 0.9 . The result indicates that with a higher furnace wall emissivity, 0.9 , the melting process is several minutes faster than that with a lower wall emissivity, 0.8. It can be concluded that it only has a small influence on the melting process. 0.8 was used in most of the simulations for the process modelling.

\section{User developed sub-models}

\section{Scrap melting sub-model with population balance}

Population Balance Model (PBM) (Sohn, et al, 1979) is a very useful tool to represent the dynamic particle size variations as function of time, distributed physical properties and other process parameters. For the application of the PBM to aluminium scrap melting, the form of PBM can be greatly simplified. It is in fact the calculation of the melting rate of the aluminium scrap at a certain position at a certain time. For a single scrap particle, it shrinks and transfers from a bigger-size group to a smaller-size group, and finally disappears. The melting rate is dependent on position, time, local temperature, local Nusselt number, scrap properties etc., and it can be calculated by the user-developed melting sub-model.

The fluid flow in the scrap-salt zone and the agitation due to furnace rotation were not fully included in the CFD based process model, and the re-solidification process was not simulated in the simplified melting sub-model and its effect on heat transfer were taken into account by some model parameters, such as the Nusselt number for the scrap melting model, and the coefficients of the effective thermal conductivity of the scrap-salt zone in the process model. Since the thermal conductivity of the aluminium metal is very high, the temperature difference within the particle can be ignored: $\partial T / \partial r=0$. When the temperature of the solid particle reaches its melting point, the heat transferred from the environment to the particle is totally used for melting of the solid metal. The heat balance at the interface between the bulk melt and the solid ( $r=R_{\text {solid }}$ ) can be expressed as follows:

$$
\rho_{p} \Delta H_{p} \frac{d R}{d t}=-h\left(T_{f}-T_{m, p}\right)
$$

where the heat transfer coefficient, $h$, can be calculated based on the particle size, fluid flow condition and bulk melt properties, and the bulk melt temperature, $T_{f}$, can be obtained from the CFD framework. Thus the size change of the particle can be calculated as follows:

$$
d R=-\frac{h\left(T_{f}-T_{m, p}\right)}{\rho_{p} \Delta H_{p}} d t
$$

For a multi-size particle system, to establish and solve the descritized PBM for aluminium scrap melting, the scrap is classified into certain groups depending on the scrap size. It can also be classified by other criteria, such as shape, composition, and scrap thermal properties, as well as the combinations of those criteria. For each cell in the scrapsalt zone, it is assumed that it has the same initial size distribution. The scrap melting sub-model was developed 
and integrated in the CFD based process model, it handles each size group the same as a single solid particle, calculates the melting rate based on the conditions in each cell in the scrap-salt zone at any time.

The melting sub-model provides the CFD framework with the information due to the melting of solid scrap, e.g. the heat sink due to melting, the amount of liquid metal and solid scrap, and the size distribution of scrap. At the same time, the CFD framework provides the information needed for the phase change calculations, e.g. the local temperatures. In this way, new particle size for each group in each cell and the total amount of melted particle in each cell, as well as the melting rate of the scrap at any position and time can be obtained. Therefore the population balance of aluminium scrap melting was established and solved by calculating the melting rates of the scrap, which was classified into some size groups. It was coupled with the CFD simulation of gas combustion, fluid flow and heat transfer in the furnace.

\section{Scrap burn-off sub-model}

Aluminium is a very reactive metal, thus oxidation is always occurring during its life. In secondary aluminium process, the oxidized aluminium can never be reclaimed in secondary aluminium process and contributes to the losses. During the melting process, aluminium scrap is sometimes exposed in a high temperature and oxidation atmosphere, despite the presence of a protecting salt layer. The generated heat amounts to $50 \%$ or more of the total energy input generated by natural gas combustion, dependent on furnace and burner operation, salt amount, scrap quality and many other factors (Boin et al., 2004). To build a valid process model, scrap burn-off must be taken into consideration.

\begin{tabular}{|c|c|c|}
\hline Group* & $\begin{array}{c}\text { Average expected } \\
\text { metal yield (\%) }\end{array}$ & Burn-off rate (\%) \\
\hline 1 & 65 & 4.42 \\
\hline 2 & 80 & 2.69 \\
\hline 3 & 90 & 2.14 \\
\hline
\end{tabular}

Group 2: granules, shredder residue, turnings

Group 3: packages (bottle caps, cooling elements, turnings), shredder residue

Table 1: Different burn-off rates for different scrap groups

Related to the distributed nature of the scrap, the scrap size distribution, surface to volume ratio, and content of contamination have a big influence on scrap burn-off. For the scrap of "good" quality, which normally has a smaller surface to volume ratio, less contaminations and/or higher metal content, the burn-off is normally less. For twentysix furnace cycles, the burn-off rate for each furnace cycle was obtained through data reconciliation by closing the mass and energy balance with minimum of standard deviation of errors. These cycles were then split into three groups based on the properties of the scrap, and the calculated results are listed in Table 1 (Salet et al.; 2003, Boin et al., 2004). Thus the total amount of scrap burn-off can be estimated. For the scrap with a metal yield of $80 \%$ (Group 2 in Table 1), the scrap burn-off is about $2.69 \%$, and the heat generated due to scrap burn-off is about 657
MJ per ton of feed. The scrap burn-off sub-model translates this part of heat into heat sources for the CFD simulations.

The current scrap burn-off model was only implemented preliminarily. The total amount of burn-off heat is estimated according to the figures in Table 1. It was assumed that the scrap burn-off heat contributes in both the gas zone and the scrap-salt zone, while the ratio of the two parts was studied as a model parameter. The burn-off reactions are assumed position independent, which is evenly distributed in each zone. The effect of burn-off on the mass balance was ignored, which is small. Kinetics involved in burn-off was not taken into account yet, however this can be assumed to be rapid. The overall heat generation rate against time was defined based on the measured off-gas temperature, which can be used to indicate the extra heat generation in the furnace.

\section{RESULTS}

\section{Gas flow and combustion in the rotary furnace}

Natural gas combustion is the main energy source for aluminium scrap melting in the rotary furnace. Its behaviour is very important to the heat transfer from the gas zone to the scrap-salt zone. In the CFD framework based on ANSYS-CFX 5.6, turbulent fluid flow, gas combustion, radiation, and conjugated heat transfer in the rotary furnace were simulated. The detailed information of the fluid flow in the gas zone, the temperature distribution in the furnace and the energy flows of the process can be obtained. As an example, Figure 4 shows the gas flow on the centre plane, from the side view $(x=0)$, in the gas zone at the $7200^{\text {th }}$ second, when about $40 \%$ of the scrap has been melted in this case. It can be noticed that, in this figure, the velocity vectors shift up at the end part of the gas zone because of the effects due to the buoyancy force and the furnace geometry. Some gas flow circles were formed in the furnace, e.g. at positions A, B and C, as shown in Figure 4.

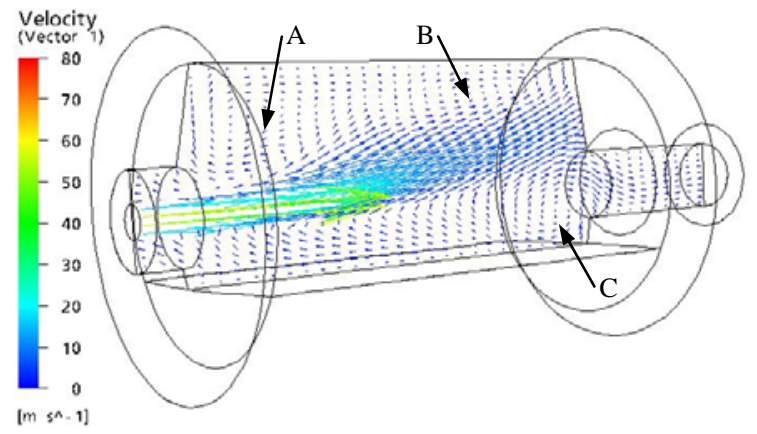

Figure 4: Flow field on the centre plane from the side view $(x=0)$ in the gas zone at $t=7200 \mathrm{~s}$.

Figure 5 and Figure 6 show the temperature field in the furnace at the $7200^{\text {th }}$ second, from the side view (on the plane where $x=0$ ) and the top view (on the plane where $y=0$ ) respectively. The flame area can be clearly seen in these figures and its temperature is as high as more than $2873 \mathrm{~K}\left(2600^{\circ} \mathrm{C}\right)$. The off-gas temperature at this moment is between $1073 \mathrm{~K}$ and $1273 \mathrm{~K}$. Figure 5 and Figure 6 also 
show the temperature in the scrap-salt zone and in the lining zone.

The average off-gas temperature at outlet can also be read in Figure 7, where the calculated values of the average off-gas temperature against the heating time are compared with the measured ones from the plant. It can be noticed that, in the beginning stage (the charging stage), the calculated values are higher than the measured ones. The reason is that during the charging stage, the leakage air through the furnace door hasn't been taken into account in the current model. In the middle, at the heating and melting stage, the two curves have a good agreement. And at the final stage (the tapping stage), the cooling process due to opening of the furnace door was not considered and only heating of the liquid metal and salt was simulated.

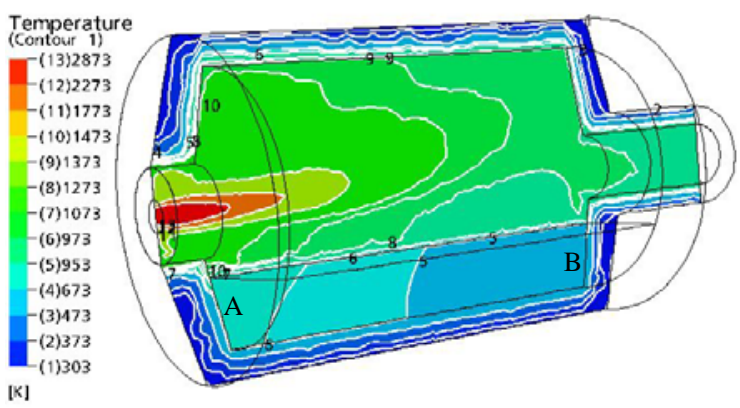

Figure 5: Temperature field on the centre plane from the side view $(x=0)$ in the furnace at $t=7200 \mathrm{~s}$.

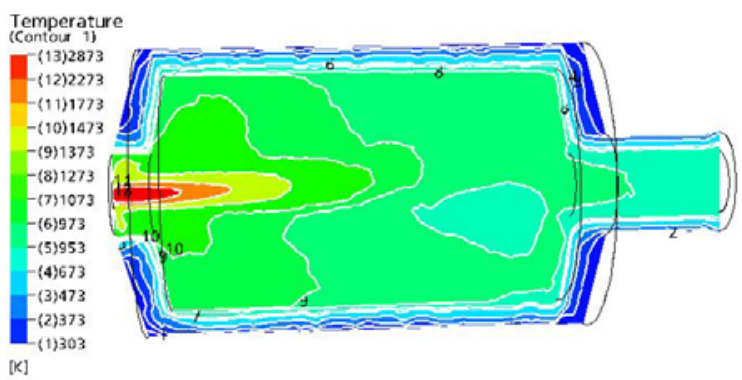

Figure 6: Temperature field on the centre plane from the top view $(y=0)$ in the furnace at $t=7200 \mathrm{~s}$.

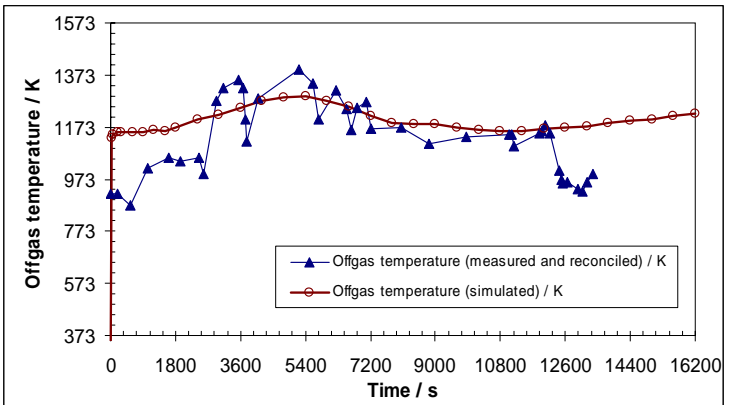

Figure 7: Simulated average temperature at outlet, compared with measured data

\section{Scrap melting in the scrap-salt zone}

One of the main purposes of this study is to obtain the total melting time under certain conditions. For example, to melt down 13 tons of scrap and 4 tons of salt flux, the simulated total melting time is about 4 hours in this case, assuming that the burn-off rate is $2.69 \%$ (about $8540 \mathrm{MJ}$ of energy generated in total) and $20 \%$ of the burn-off heat contributes in the scrap-salt zone with the other $80 \%$ in the gas zone. Figure 8 shows the changing history of the solid remaining, including the scrap and salt, against the heating time. It should be noted that the plotted remaining ratio are calculated by the total weight of the feed, which includes both scrap and salt. The melting curve can be regarded as the main criterion for the melting process, which indicates the starting and end time of the scrap melting process.

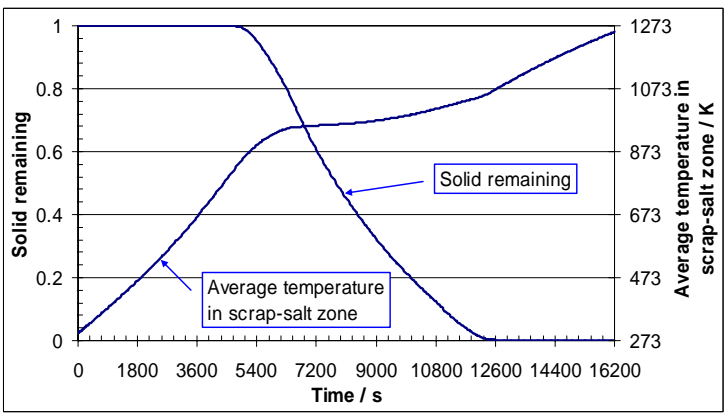

Figure 8: Melting curve of the solid (scrap and salt) in scrap-salt zone (ratio of solid remaining by total weight of feed) and average temperature in the scrap-salt zone

Figure 8 also shows the change history of the average temperature in the scrap-salt zone. During the melting stage (the middle part of the curve), when the scrap starts to melt, the increasing of the average temperature in this zone becomes slower, which is mainly due to the heat sink of the phase change. Figure 9 shows the temperature profile changing history in the centre line (point A to point $\mathrm{B}$ in Figure 5) of the scrap-salt zone. During the melting stage in the middle, the temperature difference in the scrap zone is relatively small due to the melting heat sink. The temperature difference between different parts of the scrap-salt zone can be compared with the measurements in real situation, while the data is still unavailable yet, and it can be adjusted by tuning the model parameters, such as the effective thermal conductivity coefficients, which is introduced in the previous sections.

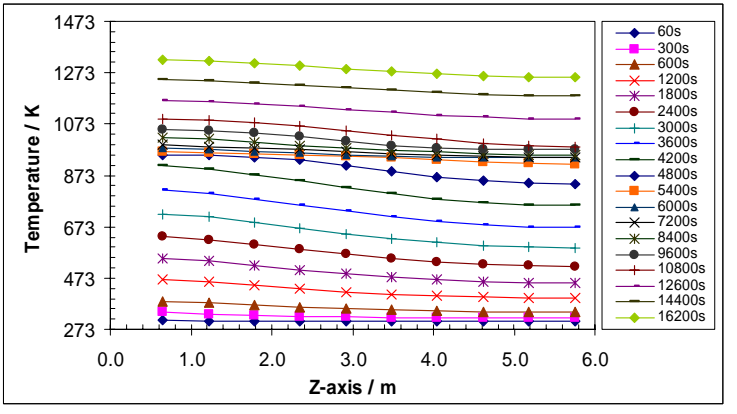

Figure 9: Temperature profile changing history in the centre line of the scrap-salt zone

\section{Energy flows and heat sources in the melting process}

For the energy balance in such a melting process, the main energy input compromises two parts: natural gas combustion heat and reaction heat due to scrap burn-off 
(oxidation). The energy input of gas combustion is about 16400 MJ for a typical cycle and the burn-off heat amounts to 8540 MJ for this case. The output of the energy compromises the following main parts: heat in liquid metal and salt slag, heat in off-gas and leakage air, and heat loss through the furnace wall.

Figure 10 shows the energy efficiency of gas combustion, which is about $60 \%$ on average for this case. Figure 10 also shows the energy output carried by off-gas, which is about $36 \%$ on average, and the heat loss through the furnace wall, which is about $4 \%$ on average. Compared to the heat carried by the off-gas, the heat loss through the furnace walls is very small. It should be noted that the burn-off heat is not included here. As introduced in the previous paragraphs, the scrap burn-off heat was taken into account by the scrap burn-off sub-model, and the predefined burn-off energy rate against time is plotted in Figure 11. It assumed that the burn-off heat contributes in both of the gas zone and the scrap-salt zone. The ratio of these two parts was studied as a model parameter, and here it's assumed that $20 \%$ of the scrap burn-off heat (1710 MJ, where the total burn-off heat is about $8540 \mathrm{MJ}$ ) contributed in the scrap-salt zone and $80 \%$ of it contributed in the gas zone (6830 MJ). Figure 11 also shows the heat sink due to scrap and salt melting (the minus sign in the figure indicates that it's a heat sink in the model). The total amount is about $7000 \mathrm{MJ}$, for melting of 13 tons of metal and 4 tons of salt in this case.

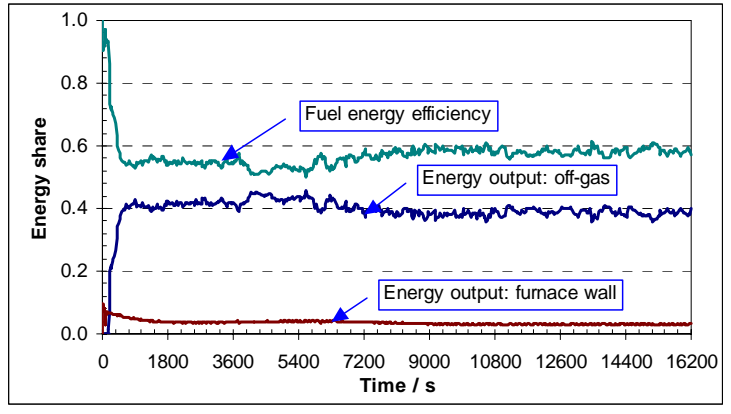

Figure 10: Overall energy flows through inlet, outlet and furnace walls

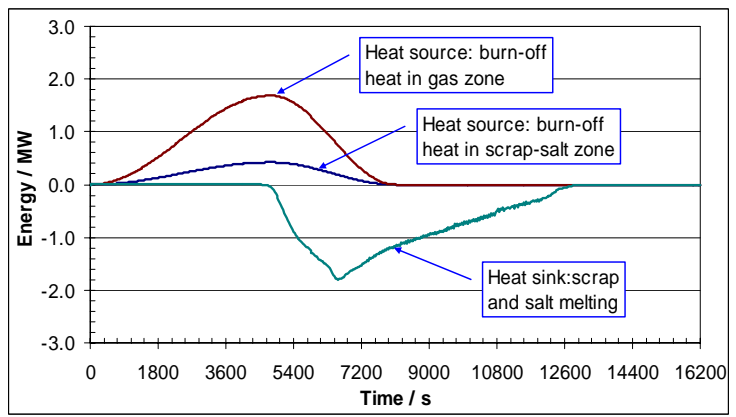

Figure 11: Heat sources due to burn-off and heat sink due to scrap and salt melting

\section{Parametric studies and case studies}

Based on the developed CFD process model, a few parametric studies and case studies were carried out, on the purpose of choosing better model parameters and process improvements. The influence of these parameters on scrap melting is briefly introduced in the following paragraphs.

\section{Scrap size and shape}

On the one hand, the experimental and numerical results (Zhou et al., 2003) suggest that an aluminium particle with a larger size needs longer time to be melted. On the other hand, if the total weight of the feed is fixed, a combination with smaller sizes of scrap has a larger total surface area, which means more salt flux is needed in operation. The influence of the scrap size distribution can be taken into account through the user-developed melting sub-model, where population balance modelling of scrap melting can be established.

The simulation result with different initial scrap size distributions shows that, for the scrap feed with larger portion of smaller particles, the melting is faster than that with larger portion of bigger particles in the earlier stage, while the total melting time is almost the same. The aluminium scrap also has a large variety of shapes, and a shape factor was applied in the melting sub-model. The result shows that it has little influence on the total melting time. It can be concluded that the melting process is mainly dependent on the heat transfer from the gas zone and the lining zone but scrap size or shape. Here it should be noted that, in reality, scrap with a larger surface to volume ratio (smaller size, more complex shape) normally has a lower metal recovery, causes a higher burn-off rate and requires more salt flux. These consequent effects have not been taken into account here, which may influence the scrap melting process.

\section{Scrap quality (scrap burn-off rate)}

Metal burn-off during the melting process is one of the main reasons of metal loss in secondary aluminium, while it also generates a large amount of heat. According to the observations and data measurements in the plant, as well as the mass and energy balance calculations, the scrap burn-off rate has an obvious relationship to the scrap type or scrap quality (Boin et al., 2004). Normally, for the "good" quality of scrap, which has a relatively small surface to volume ratio, less contamination and/or higher metal content, the metal recovery rate is higher and the metal loss is normally less.

For a total input of 13 tons of scrap feed, the scrap melting behaviour was simulated with different scrap burn-off rates, $0.0 \%, 1.5 \%, 2.0 \%, 2.7 \%$ and $3.5 \%$, and the heat generated in total is about $0 \mathrm{MJ}, 4750 \mathrm{MJ}, 6330 \mathrm{MJ}, 8540$ MJ and $11070 \mathrm{MJ}$ respectively. The total melting time is about 6.25 hours, 5.25 hours, 5.0 hours, 4.35 hours, and 3.5 hours respectively, which indicates that the total amount of burn-off has a big influence on scrap melting. A higher burn-off rate results in a shorter melting time, but it is at the expense of metal loss. The generated heat due to scrap burn-off contributes both in the gas zone and the scrap-salt zone, as discussed in the previous paragraphs, while the ratio of these two parts is difficult to decide. It was studied as a model parameter. For the same scrap burn-off rate, larger ratio in scrap-salt zone results in a faster melting of scrap, and the difference of the total melting time is within 0.5 hours. 


\section{Natural gas firing strategy}

According to the industrial observations and data measurements, the off-gas temperature at furnace outlet is much higher during the earlier stage of the process, referring to the measured off-gas temperature shown in Figure 7. The reason is mainly due to the combustion and oxidation of the organic components in scrap feed and aluminium metal itself. This part of heat contribution has been taken into account with the scrap burn-off sub-model. If we can adjust the natural gas input during this stage to adjust the total energy input, an optimised natural gas firing schedule could be obtained, and thus energy consumption could be reduced.

The result shows that if the natural gas flow rate is lowered to half of the normal input during this period, between the $3600^{\text {th }}$ second and the $8100^{\text {th }}$ second, the total melting time is about 22 minutes longer, while the total energy consumption is about $4.55 \%$ less. The adjustment of the gas firing strategy can be further optimised by refining the parameters, taking cognisance of furnace productivity and other consequent effects.

\section{Pre-heating of scrap feed}

Preheating of the scrap would reduce the total melting time, for example, if the scraps were heated to $373 \mathrm{~K}$ before charging into the furnace, the melting process is about 8 minutes shorter. And the energy consumption during the process in the rotary furnace can be reduced. While in reality, the implementation of the pre-heating facilities may be difficult and unpractical.

\section{Salt ratio in feed}

Salt flux is very important in processing of aluminium scrap in the rotary furnace. It simultaneously protects the aluminium metal from oxidation, absorbs the contamination and cleans the scrap. In industrial operation, the salt ratio in feed is estimated based on the scrap properties, e.g. scrap type, size, shape and contamination. For this case study, the salt ratio was only considered as a model parameter and cases with different salt ratios in scrap feed were simulated. The result shows that the salt ratio in feed has little effect on the total melting time. This is because of the added-up thermal effects of the salt and the aluminium metal. The salt has a higher melting point and a higher latent heat, while it has a lower heat capacity and a lower density, thus the total amount of energy needed for melting and heating of the salt or the metal is very close.

\section{CONCLUDING REMARKS}

A CFD based process model was developed for the scrap melting process in a rotary furnace. Turbulent fluid flow, conjugated heat transfer, natural gas combustion, and radiative heat transfer were simulated in the CFD framework. User sub-models, the scrap melting sub-model and the scrap burn-off sub-model, were developed and integrated into the CFD framework. A simplified population balance model for aluminium scrap melting was established by classifying the scrap feed into several scrap groups. The melting rate for each group in each cell at each time step was calculated with the exchange of information between the melting sub-model and the CFD based framework. Thus the distributed nature of the complex scrap feed can be taken into account. The distributed properties of scrap also result in distributed burn-off rates. The scrap burn-off model was developed to consider the influence of the burn-off heat on the melting process.

Based on the developed process model, behaviours of natural gas combustion, fluid flow and heat transfer in the furnace were studied, and various parametric studies and case studies were carried out focussing on the model parameter adjustment and their influence on the melting process. It shows that the scrap size and shape only have a small influence on melting, if ruling out the other consequent effects due to size and shape difference. The distributed burn-off rate, which represents the scrap quality here, is one of the important factors for the melting process. By decreasing the natural gas flow rate for some specific period, the gas consumption can be reduced, if other consequent effects can be ignored. Pre-heating of the scrap feed results in a shorter melting time and less gas consumption, while the implementation of the pre-heating facilities may be difficult and unpractical in reality. Salt ratio in feed has little influence of the total melting time, if the other consequences weren't taken into account.

\section{ACKNOWLEDGEMENTS}

This work is part of the E.E.T. (Economy, Ecology and technology, http://www.batchcentre.tudelft.nl) project supported by the Dutch government. The financial support from the E.E.T. program is gratefully acknowledged. Special thanks to Karl Konzelmann Metallschmelzwerke $\mathrm{GmbH}$, for the sharing of the information, knowledge and data, and for their support, cooperation and hospitality.

\section{REFERENCES}

Aluminium Handbook Vol. 2: Forming, Casting, Surface Treatment, Recycling and Ecology, 2003, Aluminium-Verlag, Düsseldorf.

ANSYS-CFX documentation, version CFX 5.6, ANSYS, April 2003.

BOIN, U.M.J., REUTER, M.A., and PROBST, Th., (2004), "Measuring - Modelling: Understanding the Al Scrap Melting Processes inside a Rotary Furnace”, World of metallurgy - ERZMETALL, 57, 266-271.

European Aluminium Association

http://www.aluminium.org/.

SALET, V., (2003), "Steady State Mass and Energy Balance Model of the Rotary Furnace: Study to Determine the Burn-off Rate of Aluminium Metal inside a Rotary Furnace”, MSc Thesis, Department of Applied Earth Sciences, Delft University of Technology.

SOHN, H.Y., et al, (1979), "Rate Processes of Extractive Metallurgy”, Plenum Press, New York.

ZHOU, B., YANG, Y., REUTER, M.A., (2002), “Study of Melting Behaviours of Aluminium Scraps in Molten Melts", Proc. TMS Fall 2002 Extraction and Processing Division Meeting on Recycling and Waste Treatment in Mineral and Metal Processing, Luleå, Sweden, June 1620, 527-537.

ZHOU, B., YANG, Y., REUTER, M.A., (2003), "Modelling of Melting Behaviour of Aluminium Metal in Molten Salt and Metal Bath”, Proc. Yazawa International 
Symposium on Metallurgical and Materials Processing.,San Diego, California, USA, March 3-6, 1249-1258. 